\title{
Correction to: A hybrid machine learning framework to predict mortality in paralytic ileus patients using electronic health records (EHRs)
}

\author{
Fahad Shabbir Ahmed ${ }^{1} \cdot$ Liaqat Ali $^{2,3} \cdot$ Raza UI Mustafa $^{4} \cdot$ Hasan Ali Khattak $^{5} \cdot$ Tahir Hameed $^{6} \cdot$ Iram Wajahat $^{7}$. \\ Seifedine Kadry ${ }^{8}$. Syed Ahmad Chan Bukhari ${ }^{9}$ (D)
}

(c) Springer-Verlag GmbH Germany, part of Springer Nature 2020

\section{Correction to: \\ Journal of Ambient Intelligence and Humanized Computing https://doi.org/10.1007/s12652-020-02456-3}

In the original publication of the article, the author names Fahad Shabbir Ahmad and Raza-Ul-Mustafa was incorrectly published.

The correct name should read as Fahad Shabbir Ahmad should be Fahad Shabbir Ahmed and Raza-Ul-Mustafa should be Raza Ul Mustafa.

The original article can be found online at https://doi.org/10.1007/ s12652-020-02456-3.

Fahad Shabbir Ahmed and Liaqat Ali are co-first authors.

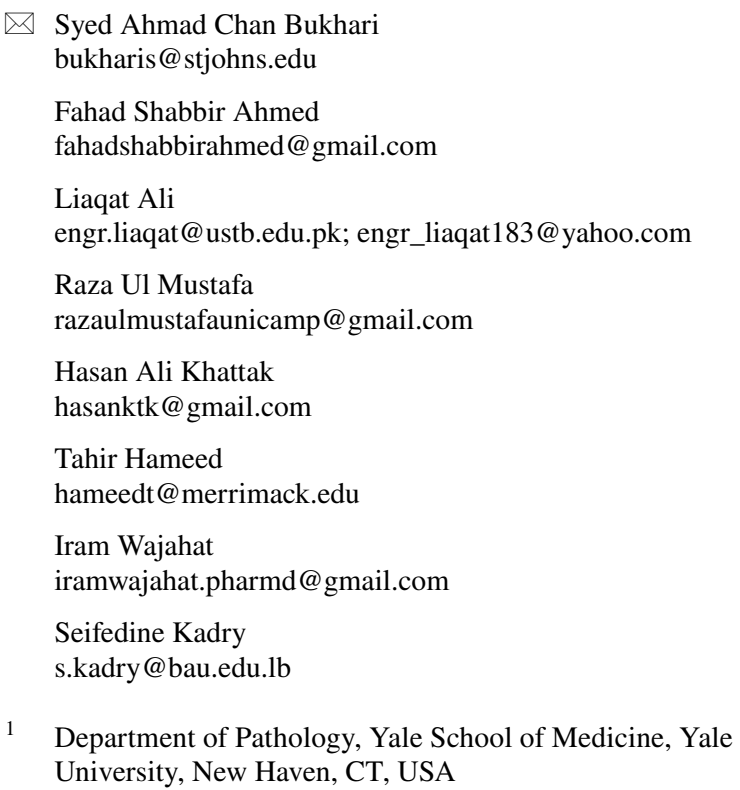

Publisher's Note Springer Nature remains neutral with regard to jurisdictional claims in published maps and institutional affiliations.
2 School of Information and Communication Engineering, University of Electronic Science and Technology of China, (UESTC), Chengdu, China

3 Department of Electrical Engineering, University of Science and Technology, Bannu, Pakistan

4 Department of Computer Engineering and Industrial Automation (DCA), School of Electrical and Computer Engineering (FEEC), Unicamp, Brazil

5 Department of Computer Science, COMSATS University Islamabad, Islamabad Campus, Islamabad 44500, Pakistan

6 Girard School of Business, Merrimack College, 315 Turnpike Street, North Andover, MA 01845, USA

7 Allied Institute of Medical Sciences, Ahmad College of Pharmacy, 50700, Gujrat, Pakistan

8 Department of Mathematics and Computer Science, Faculty of Science, Beirut Arab University, Beirut, Lebanon

9 Division of Computer Science, Mathematics and Science, Collins College of Professional Studies, St. John's University, New York, NY, USA 